\title{
CLASSIFICATION OF ROOF MATERIALS USING HYPERSPECTRAL DATA
}

\author{
C. Chisense \\ Department of Geomatics, Computer Science and Mathematics, University of Applied Sciences Stuttgart \\ Schellingstraße 24, D-70174 Stuttgart (Germany), - chembe.chisense@yahoo.com
}

KEY WORDS: Hyperspectral, Urban, ALK Vector data, Classification, Feature extraction, Roof

\begin{abstract}
:
Mapping of surface materials in urban areas using aerial imagery is a challenging task. This is because there are numerous materials present in relatively small regions. Hyperspectral data features a fine spectral resolution and thus has a significant capability for automatic identification and mapping of urban surface materials. In this study an approach for identification of roof surface materials using hyperspectral data is presented. The study is based on an urban area in Ludwigsburg, Germany, using a HyMap data set recorded during the HyMap campaign in August, 2010. Automatisierte Liegenschaftskarte (ALK) vector data with a building layer is combined with the HyMap data to limit the analysis to roofs. A spectral library for roofs is compiled based on field and image measurements. In the roof material identification process, supervised classification methods, namely spectral angle mapper and spectral information divergence and the object oriented ECHO (extraction and classification of homogeneous objects) approach are compared. In addition to the overall shape of spectral curves, position and strength of absorptions features are used to enhance material identification. The discriminant analysis feature extraction method is applied to the HyMap data in order to identify features (band combinations) suitable for discriminating between the target classes. The identified optimal features are used to create a new data set which is later classified using the ECHO classifier. The classification results with respect to material types of roofs are presented in this study. The most important results are evaluated using orthophotos, probability maps and field visits.
\end{abstract}

\section{INTRODUCTION AND RELATED RESEARCH}

Urban environments are characterized by many different artificial and natural surface materials which reflect and influence ecological, climatic and energetic conditions of cities. They include mixtures of materials ranging from concrete, wood, tiles, bitumen, metal, sand and stone. Complete inventories based on analog mapping are very expensive and time consuming. Hyperspectral data has a high spectral resolution. Therefore, it has a high potential for material oriented mapping of urban surfaces and enables the recognition of characteristic features of urban surface materials. Thus it can be expected that surface materials can be detected on a very detailed level from the hyperspectral imagery. However, the development of optimal methods for analysing hyperspectral data is still a challenge. So far there is no standard approach to material classification. Problems are the high within-class variability of many materials and the presence of numerous materials in relatively small regions. A hyperspectral pixel in an urban scene features most frequently a mixture of different material components; the classification therefore requires a decomposition of the corresponding spectral signature into its "pure" constituents (Bhaskaran and Datt, 2000).

Most of the research done on hyperspectral data in the past focused on mineral detection rather than urban surface materials such as roofs. This has changed recently due to the high pace of city development and the increase in the need to find efficient methods for mapping urban surface cover types. (Roessner et al., 2001) develop an automated method for hyperspectral image analysis exploiting the spectral and spatial information content of data in order to differentiate urban surface cover types. To achieve this, a hierarchical structure of categories is developed. The main categories are defined as sealed (buildings, roads etc.) and non-sealed surfaces (vegetation, bare soil). Similar research is carried out by (Segl et al., 2003). They analyse urban surfaces taking into account their spectral and shape characteristics in the reflective and thermal wavelength range. A new algorithm for an improved detection of pure pixels is incorporated in an approach developed for automated identification of urban surface cover types, which combines spectral classification and unmixing techniques to facilitate sensible endmember detection. (Dell'Acqua et al., 2004) investigate spatial reclassification and mathematical morphology approaches. Spectral and spatial classifiers are combined in a multiclassification framework. The use of morphological approaches gives high overall accuracies. The approach taken by (Powell et al., 2007) is similar to that adopted in the present study. They build a regionally specific (Manaus, Brazil) spectral library of urban materials based on generalized categories of urban land cover components such as vegetation and impervious surfaces. Almost $97 \%$ of the image pixels are modeled within 2.5\% RMS error constraint. The RMS error indicates the overall fit of the linear unmixing. (Heiden et al., 2007) propose a new approach for the determination and evaluation of spectral features that are robust against spectral overlap between material classes and within-class variability. The approach is divided into two parts. In the first part, spectral features for each material of interest are defined that allow an optimal identification and separation based on the reference spectra contained in the spectral library. For the second part, the robustness of these features is evaluated by a separability analysis. The results show that urban materials need to be described by more than one type of feature. Materials characterized by distinct absorption bands and/or reflectance peaks can be well detected using functions such as ratio, area, absorption depth and position. Additionally, the idea of integrating ancillary data in the analysis used by (Heldens et al., 
2008 ) is also adopted in this research. The goal of the research by (Heldens et al., 2008) is to identify urban surface materials in Munich from HyMap data. They use an unmixing approach to identify surface materials. The unmixing approach is performed with and without a building mask. The quality of the coregistration of the hyperspectral data and the building mask has a large impact on the results.

For a more comprehensive review of related work please refer to Chisense (2011).

In the present study, a material classification is performed for roofs only. The corresponding areas are identified by means of ALK vector data; hereby the confusion between roofs and roads with similar spectral properties is avoided. Information is extracted from the hyperspectral data using the "Discriminant Analysis Feature Extraction" method (DAFE). This method is used to determine an optimal set of features for further analysis, where in this particular case the term "feature" means "spectral band". Those linear combinations of bands are considered as optimal, for which the ratio of between-class variability and within-class variability is a maximum, see e.g. (Kuo and Landgrebe, 2001). The just stated variational problem leads to a generalized eigenvalue problem. A subset of transformed bands is selected depending on their corresponding eigenvalues. The new data set is classified using a spatial-spectral classifier (objectbased) known as "Extraction and Classification of Homogenous Objects" (ECHO). This method provides for a good discrimination of spectrally similar materials belonging to spatially different objects. Orthophotos, classification probability maps and field visits are used in order to evaluate the classification results. For the classification of roofs using hyperspectral data various methods have been investigated, but only those which give the most successful experimental results are discussed.

\section{STUDY AREA, HYPERSPECTRAL IMAGE DATA AND PREPROCESSING}

The hyperspectral data used in this study was acquired by German Aerospace Center (DLR) on 20th August, 2010 in the course of its annual HyMap campaign. The data covers the city of Ludwigsburg, Germany, which is located close to Stuttgart in the Neckar basin. The scene comprises six strips and extends also over adjacent rural areas, apart from Ludwigsburg city itself. The total area amounts to $11 \mathrm{~km} \mathrm{x} 16 \mathrm{~km}$. The data include a variety of typical urban structures such as residential and industrial zones, railway stations and different roads. We restricted our tests to a smaller area of approximately $2.0 \times 1.1 \mathrm{~km} 2$. The data consists of 125 bands (ranging from $0.4 \mu \mathrm{m}$ to $2.5 \mu \mathrm{m}$ ) and has a ground sample distance of $4 \mathrm{~m}$. ALK vector data with a building layer is provided by Fachbereich Stadtplanung und Vermessung der Stadt Ludwigsburg. This vector data is used for limiting the analysis to roofs. The preprocessing of the hyperspectral data was done by DLR; in particular the data was corrected for radiometric, geometric and atmospheric effects. A high resolution LiDAR surface model of Ludwigsburg with $2 \mathrm{~m}$ raster size was made available for this purpose. An overlay of the HyMap and vector data reveals a shift between the two data sets in the order of $10 \mathrm{~m}$. The vector layer for roofs is selected as the source of ground control points. The GCPs are used to georeference the HyMap data. It is observed that an overall RMS error of about 0.7 pixels is obtained after carrying out an affine transformation.

\section{METHODS AND RESULTS}

In order to determine a suitable approach, the roof material investigation is carried out in the test area before extending to the whole research area.

\subsection{Roof surface material identification}

A first look in a shop for a dealer of roofing materials already points out that a great variety of roof surface materials has to be faced in roof surface material identification. Roof tiles made from materials like clay and slate are on the market as well as those made from concrete and plastic. The widely used clay tiles are manufactured again in different ways for instance some clay tiles have waterproof glaze.

In the initial stage of the research work, 10 materials are selected for roof material identification. Three materials, namely bitumen, red roof chipping and zinc plated sheet out of the ten are identified by name from previous field visits. The remaining seven are assigned arbitrary names for identification purposes and include roof material for Kaufland shopping centre (Ludwigsburg), roof material 1, 2, 3, 4, 5 and 6. In order to map the distribution of the ten roof materials in the scene, the discriminant analysis feature extraction (DAFE) available in MultiSpec Software is applied and this is followed by classification of the data set created from the optimal features (band combinations) resulting from the feature extraction. MultiSpec is a data analysis software intended for analysis of multispectral image data or hyperspectral data. The following steps are used in order to accomplish the analysis tasks:

Selection of classes and their training sets: In order to identify and define suitable training regions, the Hymap data is classified using an unsupervised classification algorithm. The ISODATA algorithm is used for this purpose. With the aid of the output map, training regions for the 10 classes of material are defined. A class for vegetation (eleventh class) is added after it is observed that certain building parts include vegetation. Additionally, a class for the masked out area (background) is also defined.

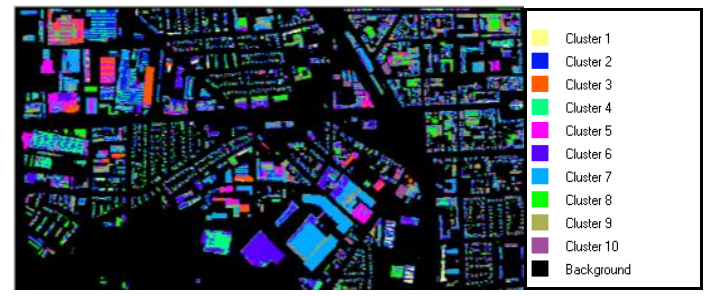

Figure 1: Output map of unsupervised classification process.

Feature extraction and classification: After designating a set of training regions, a class conditional preprocessing algorithm based on a method known as projection pursuit is performed. This algorithm does the necessary calculations in projected space rather than the original, high dimensional space thus reducing the dimensionality of the data. This is followed by the discriminant 
analysis feature extraction (determines a feature subspace that is optimal for discriminating between defined classes). The output of the extraction is a linear combination of the 125 original bands to form new bands (features) that automatically occur in descending order of their value for producing an effective discrimination. Twenty two (22) features are obtained from the feature extraction process. However, only the 11 features obtained in the final feature extraction transformation matrix (DAFE) are used to form a new data set since these provide most of the available separability and this is confirmed by the magnitude of the corresponding eigenvalues (high values). The new data is classified using the ECHO classifier. The output classification map is overlaid with an orthophoto covering the same area as shown in Figure 2.

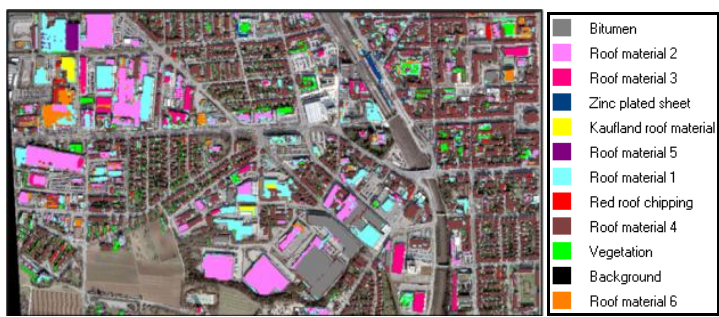

Figure 2: Overlay of classification map and orthoimage.

The classification map fits well with the orthophoto and this gives an indication of the accuracy of the classification in terms of geometry. In order to identify areas in the classification map which require improvement, the corresponding classification probability map is inspected (see Figure 3). The pixels represented by yellow to red colours in the probability map indicate a high probability of being correct. These pixels are very close to the training pixels for the classified pixels. Dark blue colours represent a low probability of being correct. The pixels represented by these colours are very far from the training pixels for all the classes and are candidates for definition of additional training regions.

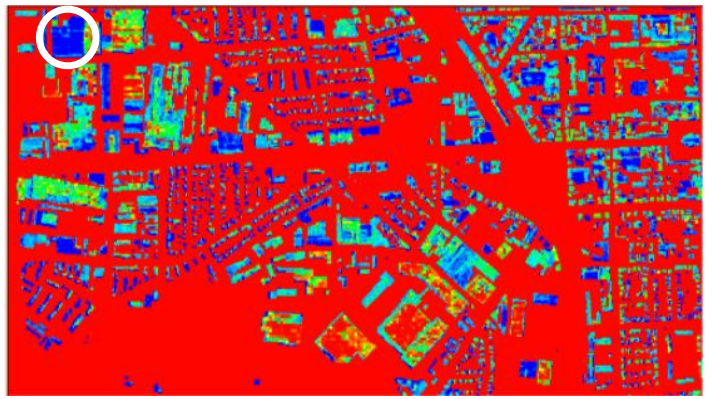

Figure 3: Classification probability map.

Defining additional training regions for areas with a low probability helps to improve the result. Most of the roofs in the probability map with a low likelihood of being correct consist of heterogeneous surface materials. For instance, the material of the roof in a white circle is not homogeneous. Therefore, additional training regions are required for areas where a surface material varies in terms of spectral properties. Defining training regions for areas requiring improvement is sufficient for achieving a classification result that represents ground features accurately. However, the required number of additional training regions depends on the scene, the material classes of interest and the accuracy requirements. The discriminant analysis feature extraction and the ECHO classifier are applied to the whole research area. The processing and analysis is done for each strip. The result obtained for each strip is shown in Figure 4.

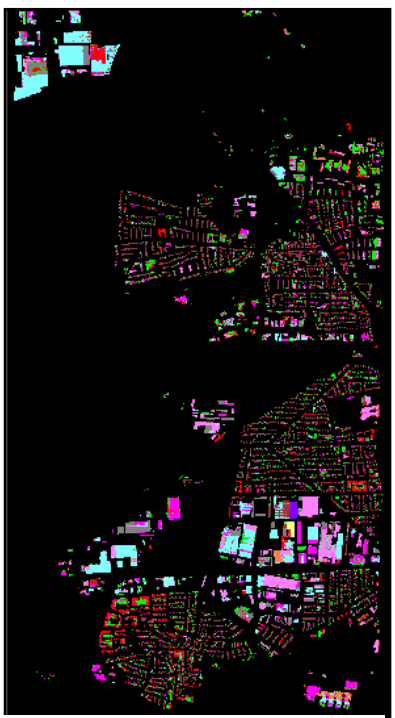

(a) Strip 1

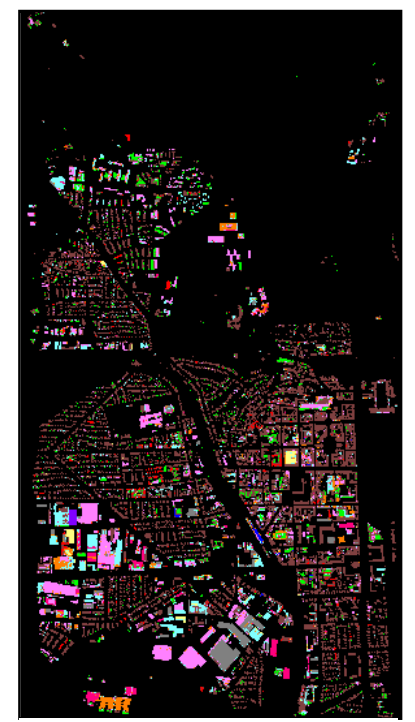

(b) Strip 2

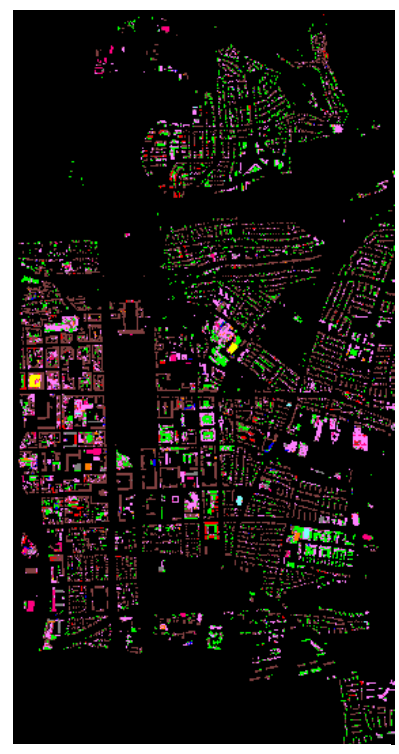

(c) Strip 3

Figure 4: Classification results of the strips covering the research area.

The output classification maps (Figure 4) fit well with orthophotos covering the research area in terms of geometry. Inspection of the corresponding classification probability maps shown in Figure 5 indicates that most of the classified building 
roofs especially in strip 1 and 2 have a high probability of being correct (red and yellow colours by default). Some of the classified building roofs especially in strip 3 have a low probability of being correct (dark blue colour by default). These building roofs mostly consist of heterogeneous surface materials. Therefore, depending on the scene, accuracy requirements and material classes of interest, more training regions should be defined for these areas and the classification process should be performed again to achieve results that represent ground features more accurately.

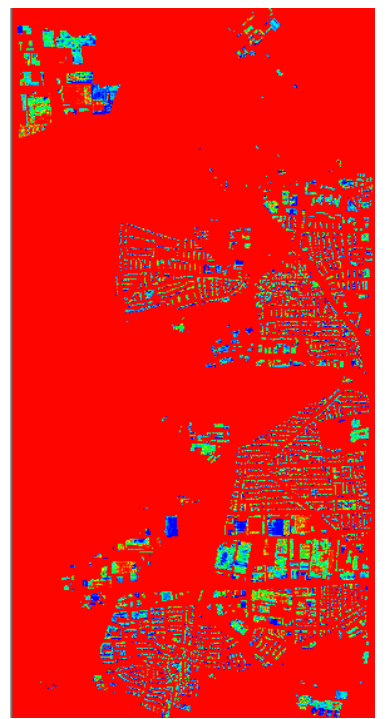

(a) Strip 1

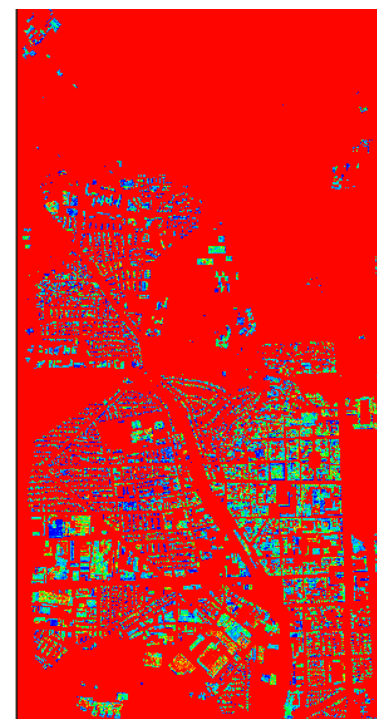

(b) Strip 2

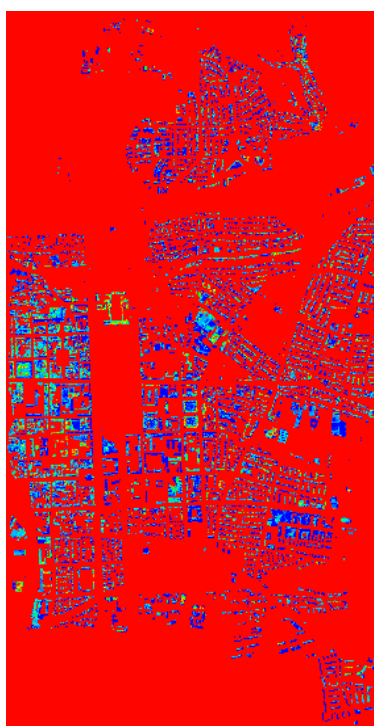

(c) Strip 3

Figure 5: Classification probability maps

The average likelihood probability of each of the strips is shown in Table 1. This indicates the degree of membership for each pixel to a particular roof material class.

\begin{tabular}{|l|l|}
\hline Strip & Average likelihood probability \\
\hline 1 & $91.4 \%$ \\
\hline 2 & $85.9 \%$ \\
\hline 3 & $88.5 \%$ \\
\hline
\end{tabular}

Table 1: Average likelihood probability

\section{CONCLUSION}

This paper focuses on the development of an approach for classification of roofs using hyperspectral data. The application of feature extraction methods such as the discriminant analysis in the identification of roofs using hyperspectral data shows good potential. In the investigation, the DAFE is combined with a spatial-spectral classifier (ECHO) to classify 10 roof materials. The ECHO classifier segments the scene into statistically homogeneous regions and then classifies the data based upon the maximum likelihood object classification scheme. The probability maps of the classification results for the test and research area show that the output classification maps have very few errors and thus confirm the success of the approach. In addition, the integration of ALK vector data for roofs in the classification process results in better discrimination of spectrally similar materials belonging to spatially different objects. This work will be continued by involving a specialist on roof surfaces (future ground truthing).

\section{REFERENCES}

Bhaskaran, S. and Datt, B., 2000. Applications of hyperspectral remote sensing in urban regions.

http://www.gisdevelopment.net/aars/acrs/2000/ps1/ps112pf.htm (24 Feb. 2011).

Chisense, C., 2011. Classification of Roofs using Hyperspectral Data. Unpublished Master thesis, University of Applied Sciences Stuttgart, Germany.

Dell'Acqua, F., Gamba, P., Ferrari, A., Palmason, J.A., Benediktsson, J.A. and Arnason, K., 2004. Exploiting Spectral and Spatial Information in Hyperspectral Urban Data with High Resolution. Geoscience and Remote Sensing Letters, IEEE, Vol. 1 , no 4, pp.322 - 326 .

Heiden, U., Segl, K., Rossner, S. and Kaufmann, H., 2007. Determination of Robust Spectral Features for Identification of Urban Surface Materials in Hyperspectral Remote Sensing Data. Remote Sensing of Environment, Vol 111, no 4, pp.537-552.

Heldens, W., Esch, T., Heiden, U. and Dech, S., 2008. Potential of hyperspectral remote sensing for characterisation of urban structure in Munich. In: Jürgens (Ed.), Remote Sensing - New Challenges of High Resolution. Proc. EARSeL Joint Workshop Bochum 2008, pp. 94-103.

Helden, W. 2010. Use of Airborne Hyperspectral Data and Height Information to Support Urban Micro Climate Characterisation. Phd thesis, Universität Würzburg, Germany. 
Powell, R.L., Roberts, A.D., Dennison, E.P. and Hess, L.L., 2007. Sub-pixel Mapping of Urban Land Cover using Multiple Endmember Spectral Mixture Analysis: Manaus, Brazil. Remote Sensing of Environment, Vol. 106, no.2 pp.253-267.

Roessner, S., Segl, K., Heiden, U. and Kaufmann, H., 2001. Automated Differentiation of Urban Surfaces Based on Airborne Hyperspectral Imagery. IEEE Transactions on Geoscience and Remote Sensing, Vol. 39, no. 7, pp.1525 - 1532.

Segl, K., Heiden, U., Mueller, M. and Kaufmann, H., 2003. Endmember detection in urban environments using hyperspectral HyMap data. Third EARSeL Workshop on Imaging Spectroscopy, Herrsching. 